\title{
Macroscopic quantum phenomena in Josephson structures
}

\author{
A. Barone ${ }^{a)}$ \\ Dip. Scienze Fisiche, Facoltá di Ingegneria, Universitá di Napoli Federico II, Piazzale Tecchio 80, \\ Napoli 80125 and CNR-SPIN, Napoli, Italy
}

F. Lombardi

Quantum Device Physics Laboratory, Dept. of Microtechnology and Nanoscience, Chalmers University of Technology, SE-412 96, Goteborg, Sweden

\section{G. Rotoli and F. Tafuri}

Dip. Ingegneria dell'Informazione, Facoltá di Ingegneria, Seconda Universitá di Napoli, via Roma 29, Aversa (CE) 81031 and CNR-SPIN Napoli, Italy

(Submitted April 13, 2010)

Fiz. Nizk. Temp. 36, 1098-1106 (October-November 2010)

The Josephson effect is a probe with unparalleled capabilities for the study of a variety of macroscopic quantum phenomena. This is a survey of important achievements and challenging trends, in particular macroscopic quantum tunneling and energy level quantization. We focus on high- $T_{C}$ superconducting structures and recent research on nanostructures. (C) 2010 American Institute of Physics. [doi:10.1063/1.3517171]

\section{INTRODUCTION}

The importance of the Josephson effect ${ }^{1-5}$ is widely recognized both for its intrinsic relevance in the context of superconductivity and for the large variety of its actual and potential applications. The Josephson effect still plays a fundamental role in various challenging topics such as that of a powerful probe of the symmetry of the order parameter characterizing different classes of superconductive materials. Several aspects of the Josephson effect are of paramount importance in the context of macroscopic quantum phenomena. Among these, quantum decay via macroscopic quantum tunneling (MQT) merits great attention, as does energy level quantization (ELQ) and other phenomena intrinsic to quantum mechanics at a macroscopic level, such as macroscopic quantum coherence in a superconducting quantum interference devices (SQUID). In addition, weakly coupled BoseEinstein condensate (BEC) systems are now the subject of thorough theoretical and experimental studies relating to interference phenomena and the Josephson effect. ${ }^{6}$

The origin of superconductivity in oxide compounds is still somewhat mysterious. The phenomenon of HTS encompasses a wide range of interesting issues at the frontier of our understanding of solid state systems and at the limit of existing techniques in materials science and nano-technology. Josephson junctions have played an indispensable role in determining the crucial properties of HTS. $d$-wave order parameter symmetry (OPS) is probably the most remarkable example. ${ }^{7,8}$ Imagine the situation a few months before the discovery of HTS. Who would have supposed that in a few months a supercurrent would flow at a temperature of about $100 \mathrm{~K}$ ? Who would have imagined a supercurrent between two phase coherent electrodes up to about $100 \mathrm{~K}$ ? What about the thermal energy, the gap value, the Josephson coupling energy, the charging energy, the coherence length, the critical stoichiometry, and so on? These considerations lead to the first obvious feature, which is independent of the still mysterious origin of superconductivity in HTS, of their very complicated structure, and so on: oxides extend superconductivity to unexpected energy and length scales. In this short review we try to explore new features of HTS by means of the Josephson effect, with special attention to macroscopic quantum phenomena and to mesoscopic effects.

\section{THERMAL AND MACROSCOPIC ACTIVATION OF QUANTUM TUNNELING}

Quantum tunnelling on a macroscopic scale was considered by Sidney Coleman ${ }^{9}$ in the context of ground state metastability in a cosmological framework. The false vacuum, was interpreted as decaying through barrier penetration, toward a true vacuum, a more stable state of the Universe. In the Josephson junction-cosmology analogy, the macroscopic degree of freedom is the relative phase, $\varphi$, between the two weakly coupled superconductors (or the trapped magnetic flux, $\Phi$, in an rf SQUID superconducting loop). We consider a potential

$$
U=-\Phi_{o} /(2 \pi)\left(I_{C O} \cos (\phi)+I \phi\right)
$$

(in Fig. 1 a small section of the washboard potential is shown to focus on a single period of the periodic structure; this is used below to formulate the macroscopic quantum tunneling problem) given by the sum of the free energy associated to the Josephson junction barrier and a linear term in $\varphi$ owing to a bias current $I$. $I_{C O}$ represents the maximum Josephson current. This potential can be also easily derived from the a resistively and capacitively shunted junction (RCSJ) model applied to a Josephson junction. The Josephson inductance $L_{J}$ and capacitance $C$ act as an anharmonic LC resonator (at zero voltage) with resonance frequency $\omega_{P}=\left(L_{J} C\right)^{-1 / 2}$ (plasma frequency), where $L_{J}=\Phi_{O} /\left(2 \pi I_{C O} \cos (\phi)\right)=\Phi_{O} /$ $\left(2 \pi\left[I_{C O}^{2}-I^{2}\right]\right)^{1 / 2}$ and $\left.\omega_{P}=\left(2 \pi I_{C O} /\left(\Phi_{O} C\right)\right) /\left(1-\left(I / I_{C O}\right)^{2}\right)\right)^{1 / 4}$. Representing the displacement current by a capacitor $(\mathrm{C})$ and the sum of the quasiparticle and insulator leakage current by 


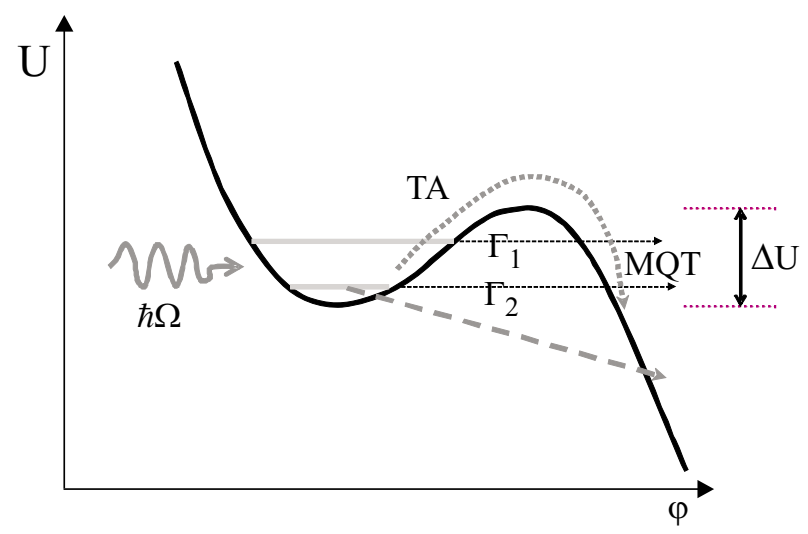

FIG. 1. Detail of a washboard potential in the RCSJ model for a finite bias. $\Delta \mathrm{U}$ represents the energy barrier. $\mathrm{T}_{\mathrm{A}}$ denotes thermal activation (dotted line), and MQT processes with low (solid line) and high (dashed line) dissipation, ${ }^{15}$ from the ground state and the first excited state, respectively, are indicated. Microwaves can induce a transition from the ground to the first exited state.

a resistance $(R)$, we obtain an equivalent circuit for the junction:

$$
I+I_{N}=I_{C O} \sin (\phi)+V / R+C d V / d t .
$$

The second term contains the well-known dc Josephson equation $I_{C}=I_{C O} \sin (\varphi)$. The noise source $I_{N}$ is associated with its shunt resistance.

In the mechanical analogy of this problem we can refer to a particle of mass $m=\left(\Phi_{O} / 2 \pi\right)^{2} C$ in such a washboard potential (see Eq. (1)) and specify two states corresponding to the particle at rest ( $\varphi$ constant) or running down the slope ( $\varphi$ time-dependent). The motion of the particle is subject to damping given by $1 / Q$, where $Q=\omega_{P} R C$ is the quality factor. Thus, in terms of the constitutive Josephson effect relations, these states will correspond to the zero voltage and a finite voltage state, respectively, in the current-voltage (I-V) characteristics. At zero temperature these transitions will occur as soon as the average slope of $U(\varphi)$ rises to a value such that there are no valleys (i.e., when the current bias reaches $I_{C}$ ), so that the particle can run down the slope. That is, $\varphi$ becomes time-dependent and a transition to the finite voltage state of the $I-V$ curve occurs.

Depending on the mechanism of dissipation, conditions ranging from overdamping with single-valued $I-V$ curves to underdamping generating highly hysteretic $I-V$ curves can occur. In the former case, large dissipation will restore the $V=0$ state as soon as the current bias is lowered to the critical value while, in the underdamped regime, the effect of the junction capacitance dominates the dissipation. The mechanical analogy, is obvious with the interplay between the friction and inertial mass of the particle.

In the pure thermal regime, the rate of the transition from weak to moderate damping $(Q>1)$ is determined by the original Kramer theory as

$$
\Gamma_{t}=A \frac{\omega_{P}}{2 \pi} \exp \left(-\frac{\Delta U}{k_{B} T}\right)
$$

where $\Delta U(a)=(4 \sqrt{2} / 3) E_{J}(1-\gamma)^{3 / 2}$ is the barrier height and is illustrated in Fig. 1 for $\gamma=I / I_{C 0}$ close to 1 , with $E_{J}$ $=I_{C O} \Phi_{0} / 2 \pi$. The prefactor $A$ can be specified for various damping regimes. The transition rate will be dominated by MQT at low enough temperature: ${ }^{11,12}$ for $Q>1$ and $\gamma$ close to 1 it is approximated by the expression for a cubic potential:

$$
\Gamma_{q}=a_{q} \frac{\omega_{P}}{2 \pi} \exp \left[-\frac{\Delta U}{\hbar \omega_{P}}\left(1+\frac{0.87}{Q}\right)\right),
$$

where $a_{q}=\left[864 \pi \Delta U / \hbar \omega_{P}\right]^{1 / 2}$. The occurrence quantum activation of this sort in Josephson junctions confirms the validity of quantum mechanics at a macroscopic level for a macroscopic variable, namely the relative phase $\varphi$. The complementary quantum phenomenon is quantized energy levels (ELQ). Evidence of this effect is provided by experiments based on microwave irradiation with consequent energy level hopping.

Macroscopic quantum tunnelling in the context of Josephson structures was proposed by Anderson, ${ }^{2}$ Ivanchenko and Zilberman ${ }^{11}$ and Caldeira and Leggett, ${ }^{12}$ who gave a quite complete description introducing the fundamental aspect of the effect of dissipation. The crossover temperature between the thermal and the quantum regimes is $T_{\text {cr }}$ $=\left(\hbar \omega_{P} / 2 \pi k_{B}\right)\left[\left(1+1 / 4 Q^{2}\right)^{1 / 2}-1 / 2 Q\right] \cdot{ }^{13}$ Below $T_{\text {cr }}$ quantum effects are dominant over thermal effects. ${ }^{12,14}$ A variety of successful experimental observations of MQT have been made and these confirm the effect of dissipation in reducing the decay rate by quantum activation. ${ }^{16,18}$ Clear evidence of the transition from thermal to quantum activation was obtained in pioneering experiments to measure the decay rate, which decreases with the temperature down to $T_{\mathrm{cr}}$, while a temperature independent activation prevails for $\mathrm{T}<\mathrm{T}_{\mathrm{cr}}$. For an excellent description of experiments in this context the reader is referred to a paper by Clarke et al. ${ }^{19}$ Another issue of interest is the phenomenon of resonant macroscopic quantum tunneling $(\mathrm{RMQT})^{20}$ resulting in the occurrence of sharp voltage peaks due to a MQT process between levels in neighboring wells with close energies. This effect has been experimentally confirmed by Rouse, Han, and Lukens ${ }^{21}$ in a SQUID. As for macroscopic quantum coherence (MQC), examples of relevant proposals and experiments can be found in Refs. 22-24.

\section{MACROSCOPIC QUANTUM TUNNELING IN HTS}

In recent years, interest in superconducting quantum devices has expanded to high temperature superconductors (HTS), especially in view of the possible advantages of $d$-wave OPS ${ }^{7,8}$ for quiet qubits. ${ }^{25}$ This implies the possibility of building so-called $\pi$-junction devices. The local magnetization in $\pi$-loops, i.e., loops formed with an odd number of $\pi$-junctions, could serve as states in a qubit device. The main advantage of such an unconventional qubit device is that it works without an external field bias.

These properties of HTS devices could be also related to the search for a "protected qubit". This last can be traced to the seminal work of Kitaev ${ }^{26}$ and has been applied to superconducting qubits by Ioffe et al. ${ }^{25,27}$ The basic idea is that a topological object, say a magnetic "flux" configuration over an array of Josephson junctions, which could take place at a classical level before quantum effects came into play, has the property of being insensitive to some perturbations which are topological invariants of the system. 
The use of topology for making a robust qubit, i.e., one that is insensitive to its surroundings, can be found in the experiments by Wallraff et $a l^{28}$ They have also shown that fluxons in annular Josephson junctions can behave as quantum objects at low temperatures and could, in principle, be used as qubits when subjected to a magnetic field-induced potential. $^{29}$

HTS may be an interesting starting point for novel ideas on key issues in coherence and dissipation in solid state systems because of their unusual properties, in particular the presence of low energy quasi-particles due to nodes in the $d$-wave OPS. ${ }^{30,31}$ Since the very beginning this has been a strong argument against the occurrence of macroscopic quantum effects in these materials. Quantum tunnelling of the phase leads to a fluctuating voltage across the junctions which excites the low energy quasi-particles specific to $d$-wave junctions, causing decoherence. Contributions to dissipation from different transport processes, such as channels due to nodal quasiparticles, midgap states, or their combination, have been identified. ${ }^{32-35}$ In particular cases, decoherence times and quality factors were calculated assuming a system coupled to an Ohmic heat bath. It has also been argued that difficulties in observing quantum effects owing to the presence of gapless quasi-particle excitations can be overcome by choosing the proper working phase point. ${ }^{33}$ In particular, decoherence mechanisms can be reduced by selecting appropriate tunnelling directions because of the strong phase dependence of the quasiparticle conductance in $d$-wave GB junctions.

Searching for macroscopic quantum effects become possible once high quality HTS Josephson junctions ${ }^{36,37}$ with significant hysteresis in their current-voltage characteristics were available. We can distinguish two classes of experiments based on two different complementary types of junctions: 1) MQT and ELQ, ${ }^{30,31}$ on off-axis YBCO grain boundary (GB) biepitaxial JJs, where experiments are designed to study $d$-wave effects with a lobe of one electrode facing a node of the other; 2) MQT and ELQ on intrinsic junctions of single crystals of different materials, ${ }^{38,39}$ where $d$-waves are expected to play a minor role. ${ }^{33,34}$ Experiments using GBs are more complicated because of the complexity of these junctions, but are very complete and can be used to examine the effects of a $d$-wave OPS on dissipation and coherence. Only GB junctions can be more easily integrated into circuits.

The GB biepitaxial junctions ${ }^{40,41}$ used in Refs. 30 and 31 had reproducible hysteretic behavior up to $90 \%$. One particular feature of these structures is the use of a (110)-oriented $\mathrm{CeO}_{2}$ buffer layer, deposited on (110) $\mathrm{SrTiO}_{3}$ substrates. YBCO grows along the [001] direction on the $\mathrm{CeO}_{2}$ seed layer, while it grows along the $[103] /[013]$ direction on $\mathrm{SrTiO}_{3}$ substrates. ${ }^{41,42}$ The $\mathrm{CeO}_{2}$ induces an additional $45^{\circ}$ in-plane rotation of the $\mathrm{YBCO}$ axes with respect to the inplane directions of the substrate (Fig. 2a). Atomically flat interfaces can be achieved under appropriate conditions. ${ }^{40} \mathrm{As}$ a consequence, the GBs are the product of two $45^{\circ}$ rotations, a first one around the $c$-axis, and a second one around the $b$-axis. This configuration produces a $45^{\circ}$ misorientation between the two electrodes to enhance $d$-wave order parameter effects by varying the interface orientation.
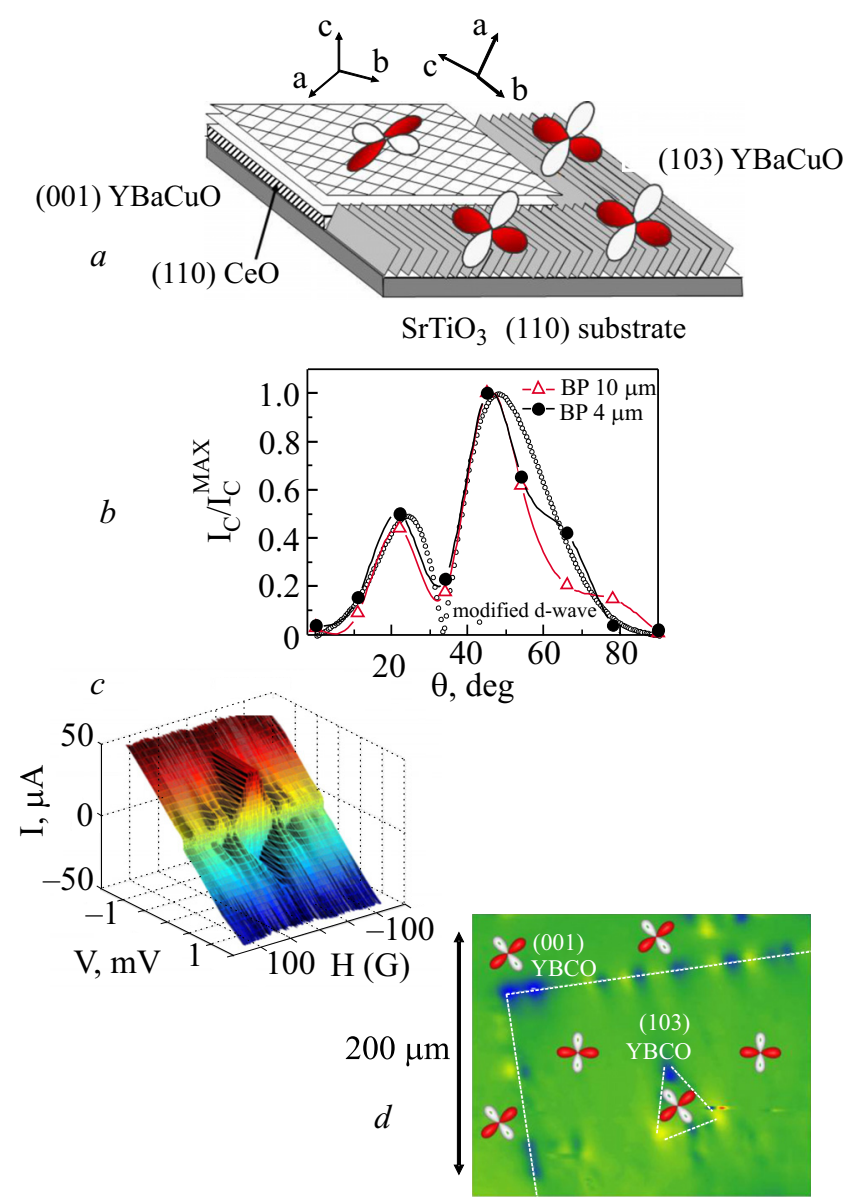

FIG. 2. (a) Sketch of the grain boundary structures in biepitaxial $\mathrm{CeO}_{2}$ (b)-based out-of-plane biepitaxial junctions. The $\mathrm{CeO}_{2}$ produces an additional $45^{\circ}$ in-plane rotation of the YBCO axes with respect to the in-plane directions of the substrate. (b) Normalized critical current density $\mathrm{J}_{\mathrm{C}} v s$. angle $\theta$ for two sets of c-axis tilt biepitaxial YBCO junctions, with widths of $10 \mu \mathrm{m}$ (triangles) and $4 \mu \mathrm{m}$ (stars). The solid curves connecting the symbols are guides for the eye. The dotted curve is the Sigrist-Rice-like formula assuming pure $d_{x^{2}-y^{2}}$ pairing symmetry in this geometry. ${ }^{41}$ (c) I-V curves as a function of the magnetic field. A Fraunhofer profile of the critical current can be seen. The misorientation angle is $60^{\circ}$ in this case. (d) Scanning SQUID microscope image of a $200 \times 200 \mu \mathrm{m}^{2}$ area, including tilt-tilt and twist-tilt in $\mathrm{CeO}_{2}$-based biepitaxial GBs. The GBs are marked by the presence of spontaneous currents. The sample was cooled and imaged at $\mathrm{T}$ $=4.2 \mathrm{~K}$ in nominally zero field. Adapted from Ref. 43 .

In addition, the possibility of tuning the critical current $I_{C}$ by varying the interface orientation $\theta$, in complete agreement with the predictions of a $d$-wave OPS (see Fig. 2b), ${ }^{41}$ makes it possible to adjust the junction for an MQT experiment when the OPS configuration is known exactly. Specific angle orientations can favor both junctions with a Fraunhofer-like pattern (Fig. 2c) and the spontaneous generation of fractional vortices (Fig. 2d). ${ }^{43,37}$ A suitable junction can be, therefore, selected for an experiment. Since interest has mostly focused in those features that are distinct from the case of low $T_{c}$ superconductor (LTS) junctions, namely effects due to OPS and dissipation due to low energy quasiparticles, the junction in the tilt configuration (angle $\theta=0^{\circ}$ ) turns out to be the most interesting case for MQT and ELQ experiments. This configuration (lobe to node) maximizes $d$-wave induced effects and makes it possible to explore the effects of low energy quasiparticles. 


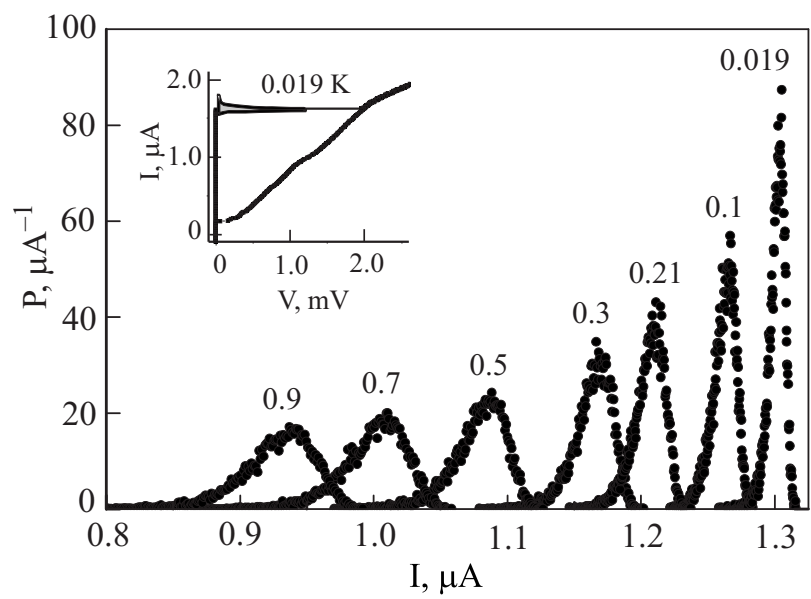

FIG. 3. Switching current probability distribution for $\mathrm{I}_{\mathrm{C} 0}=1.40 \mu \mathrm{A}$ at $\mathrm{B}$ $=0 \mathrm{~T}$ for different bath temperatures $\mathrm{T}_{\text {bath. }}$. The inset shows the switching current probability distribution measured at $\mathrm{T}=0.019 \mathrm{~K}$ along with the original I-V curve. Adapted from Bauch et al. ${ }^{30}$

Some new features of Josephson dynamics could be accessible in HTS junction configurations, for example, the role of Andreev bound states ${ }^{44}$ and intrinsic doubly degenerate fundamental states. ${ }^{36,37}$ The latter are the result of an unconventional Josephson current-phase relation (CPR) which reveals the presence of higher harmonics $(\sin 2 \varphi)$ owing to the $d$-wave symmetry of the order parameter. ${ }^{45}$ The dynamics of a current biased JJ also strongly depends on the CPR. The features of junctions that responsible for the $\sin 2 \varphi$ component, not yet been identified unambiguously in systems characterized by faceting of the grain boundary line. ${ }^{45}$ A detailed description of the features of a JJ with both first and second harmonic components in the CPR (we neglect higher harmonics due to the low junction barrier transparency) is beyond the scope of this review. ${ }^{30,46}$

\section{A. Experiments on YBCO biepitaxial Josephson junctions}

We follow Refs. 30 and 31 in reporting the first experimental measurements on MQT in HTS $J J_{\mathrm{s}}$; details can be found in those papers. The transition rate of the superconducting phase $\varphi$ from a local minimum in the washboard potential to the moving state as a function of temperature has been investigated by analogy with experiments on low- $T_{c}$ junctions. Figure 3 shows a set of switching current probability distributions as functions of temperature for an biepitaxial $J J$. The inset of Fig. 3 shows the switching current probability distribution measured at $T=0.019 \mathrm{~K}$, along with the original $I-V$ curve.

The measured $\sigma$ saturates below $50 \mathrm{mK}$, indicating a crossover from the thermal to the MQT regime. An estimate of $R \simeq 100 \Omega$ for the electrode impedance is obtained from a microstrip transmission line model and $C_{S} \simeq 1.6 \mathrm{pF}$ (which is not far from the rough estimate of $C$ obtained from the hysteresis in the dc- $I-V$ curve $\left.^{47}\right)$. An estimate of $C_{J}$ can be obtained by using Eq. (2) in the MQT regime. ${ }^{48}$ The resulting value of $C_{J} \sim 0.22 \mathrm{pF}$ gives a plasma frequency of $\omega_{P} / 2 \pi \simeq 2.6 \mathrm{GHz}$ and a quality factor greater than 1 in the quantum regime. The observed crossover temperature $(T$

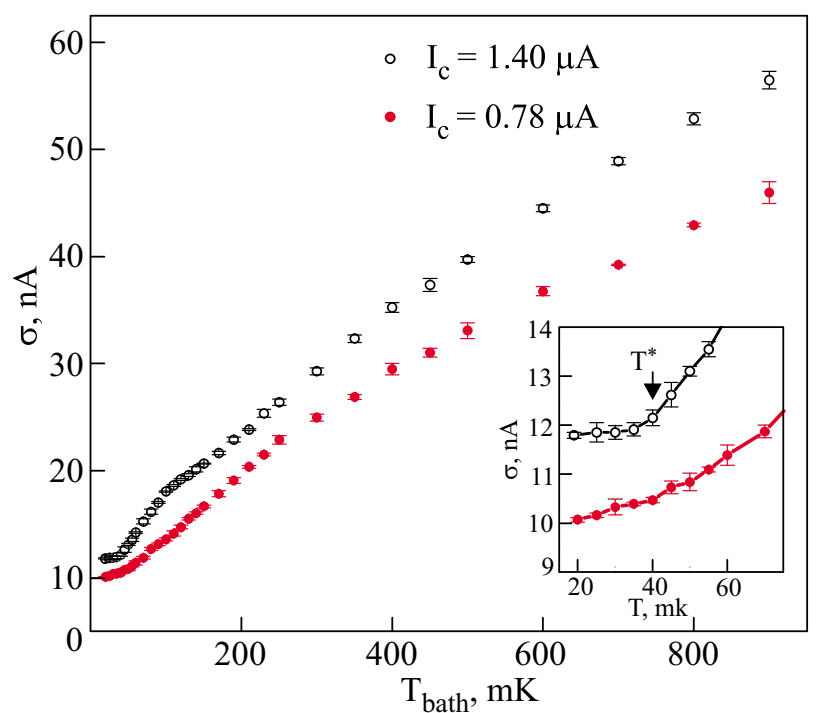

FIG. 4. The measured $\sigma$ saturates below $50 \mathrm{mK}$, indicating a crossover from the thermal to the quantum regime. The width $\sigma$ for $\mathrm{B}=2 \mathrm{mT}$ and the data for $\mathrm{B}=0 \mathrm{mT}$ are shown in the inset. Adapted from Bauch et al. ${ }^{30}$

$\simeq 50 \mathrm{mK}$ ) between the thermal and the quantum regimes is consistent with the predicted values from $T_{c}^{1 D}$ $\simeq\left(\hbar \omega_{P} / 2 \pi k_{B}\right) .^{13,48}$

To exclude the possibility that the saturation of $\sigma$ is due to some spurious noise or heating in the measurement setup, the switching current probability distributions were measured at a reduced critical current $\left(I_{C 0}=0.78 \mathrm{~A}\right)$ by applying an external magnetic field $B=2 \mathrm{mT}$. The width for $B$ $=2 \mathrm{mT}$ is plotted in the inset of Fig. 4. The data in the presence of a magnetic field clearly show a smaller width, which does not saturate down to the base temperature. At temperatures between 50 and $100 \mathrm{mK}$ the data start to follow the well known $\sigma^{2 / 3}$ dependence owing to thermal transitions ${ }^{49}$ (see the dashed line in Fig. 4). However, at $T \sim 110 \mathrm{mK}$ there is an hump, i.e., a transition to a $\sigma^{2 / 3}$ dependence with lower values (solid line). The higher values t low temperatures correspond to an enhanced thermal transition rate. One possible explanation of this effect is the onset of a second harmonic component in the CPR at low temperatures owing to low transparency of the junction barrier. ${ }^{30}$

Aside from being one of the keys to low barrier transparency, another important consequence of $c$-axis tilt is the existence of a significant kinetic inductance in the model of a YBCO JJ. Indeed, in these junctions, the kinetic inductance and stray capacitance control the main difference in the washboard potential that makes the system behavior depend on two degrees of freedom. ${ }^{31}$ The YBCO $J J$ is coupled to this $L C$-circuit (Fig. 5a) and the potential becomes twodimensional (2D). Similar behavior has been observed in a low- $T_{C}$ dc superconducting quantum interference device. ${ }^{50}$

The LC coupling can be described by the following 2D potential: ${ }^{48,51}$

$$
U\left(\phi, \phi_{S}\right) / E_{J}=\frac{1}{2 \beta}\left(\phi_{j}-\phi_{S}\right)^{2}-\gamma \phi_{S}-\cos \phi_{j},
$$

where $\phi_{S}=\phi_{j}+\left(2 \pi / \Phi_{0}\right) I_{S} L_{S}$ is the phase difference across the shunt capacitor $C_{S}$ and $I_{S}$ the current in the inductance $L_{S}$. It has been shown that the barrier height $\Delta U$ is the same as in 


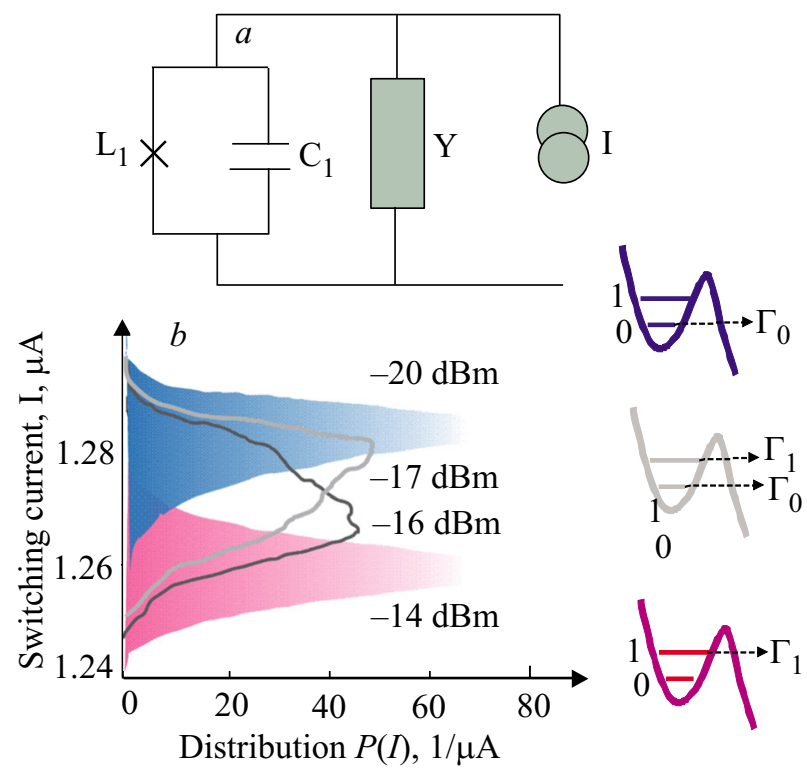

FIG. 5. The measured switching current probability distribution $\mathrm{P}(\mathrm{I})$ in the presence of microwaves at a frequency of $850 \mathrm{MHz}$ and at a temperature $\mathrm{T}=15 \mathrm{mK}$. The applied power at the room temperature terminal varies from -20 to $-14 \mathrm{dBm}$. The MQT processes corresponding to the switching current probability distribution $\mathrm{P}(\mathrm{I})$ are shown on the right. Adapted from Bauch et al. ${ }^{31}$

the $1 \mathrm{D}$ case. $^{51,48}$ The $2 \mathrm{D}$ potential modifies the value of the crossover temperature and, in general, of both the thermal and MQT transition rates. In particular, in an experiment ${ }^{31}$ the $L C$ values were $L_{S} \simeq 7.2\left(\Phi_{0} / 2 \pi I_{C O}=7.2 L_{J} 0\right)$ and $C_{S}$ $\simeq 7.2 C_{J}$.

By analogy with the LTS case, the normalized bias current $\gamma$ is ramped from zero to a value near to 1 , at finite temperature; the junction may switch into a finite voltage state for a bias current $<1$. This corresponds to a particle escaping from the well either by a thermally activated process or by tunneling through the barrier potential (MQT). In the pure thermal regime, the transition rate for weak to moderate damping $(Q>1)$ is given by

$$
\Gamma_{t}=a_{t}^{2 D} \frac{\omega_{R}}{2 \pi} \exp \left(-\frac{\Delta U}{k_{B} T}\right),
$$

where $\Delta U=(4 \sqrt{2} / 3) E_{J}(1-\gamma)^{3 / 2}$ is the barrier height for $\gamma$ close to 1 , and $\omega_{R}$ is the attempt frequency in the well. Explicit expression for 2D thermal prefactor $a_{t}^{2 D}$ can be found in Ref. 48. In the limit of large $L_{S}$ and $C_{S}$ (as in the experiment of Ref. 31), this correction is small and is mainly due to a shift in the attempt frequency, which is lower than the standard $J J$ plasma frequency $\omega_{P}$.

The transition rate will be dominated by MQT at temperatures low enough for $Q>1$, where the expression for the $2 \mathrm{D}$ potential is:

$$
\Gamma_{q}=a_{q}^{2 D} \frac{\omega_{P}}{2 \pi} \exp \left[-\frac{36 \Delta U}{5 \hbar \omega_{P}}\left(1+\frac{5 L_{J}}{2 L_{S}}\right)\right],
$$

where $a_{q}=\left[864 \pi\left(\Delta U / \hbar \omega_{P}\right)\left(1+5 L_{J} / 2 L_{S}\right)\right]^{1 / 2}$, so the MQT rate is reduced by a factor of $5 L_{J} / 2 L_{S}$. ${ }^{48}$ Equations (1) and (2) also yield an expression for $\sigma(T)$; the FWHM of the switch distribution $\mathrm{P}(\mathrm{I})$ can be numerically calculated and compared with experiments.
The theory reported in Ref. 48 is in excellent agreement with experimental transition rates, though, at the moment, it cannot explain the hump structure of $\sigma$ near $0.1 \mathrm{~K}$. The problem may be related to a correct description of dynamical/ thermal population of excited states in the metastable well, which is neglected in the $L C$-circuit model. ${ }^{48}$

The $L C$-circuit model, the so-called "shell" circuit, is also of great importance for explaining the energy level quantization (ELQ) experiment reported in Ref. 31. Microwaves at frequency $\omega_{r f}$ were transmitted to a junction via a simple dipole antenna at a temperature below $T_{c}$. When $\omega_{r f}$ of the incident radiation (or multiples of it) coincides with the bias current-dependent level separation of the junction, $\nu_{10}(\gamma)=m \omega_{r f}$, the first excited state is populated. Here, $m$ is an integer corresponding to an $m$-photon transition from the ground state to the first excited state. Fig. 5b shows the evolution of the switching-current histogram as a function of applied microwave power for an $m=3$ three-photon process.

At lower powers $(-20 \mathrm{dBm})$, the transition is basically from the ground state, since the occupation probability for the first excited state is negligible. When the applied power is increased $(-17 \mathrm{dBm}$ and $-16 \mathrm{dBm})$, the first excited state begins to be populated. Then two peaks appear in the histogram corresponding to tunneling from both the first excited $\Gamma_{1}$ and ground $\Gamma_{0}$ states. The transition from the first excited state is exponentially faster and dominates, and the switching current distribution is again single peaked at $-14 \mathrm{dBm}$. The Lorentzian-shape of the transition rate implies a $Q$ value on the order of $40,{ }^{31}$ comparable to the initial best results obtained for LTS junctions.

Specific effects related to stray capacitance and large kinetic inductance were discussed in the original paper ${ }^{31}$ and subsequently. $^{48}$

The observations of quantum tunneling, narrow width of excited states, and large $Q$ support the notion of "quiet" qubits based on $d$-wave symmetry of the superconductor, but the significance of the experiments goes beyond this. There may be some mechanism preventing low-lying quasiparticles in the $d$-wave state from causing excessive dissipation. There may also be some kind of condensation mechanism for quasiparticles, in general agreement with the HTS SU(2) slaveboson model, where the physical properties of the low lying quasiparticles are found to resemble those in BCS theory. ${ }^{52}$ The existence of a subdominant imaginary $s$-wave component of the order parameter inducing a gapped excitation spectrum could be another possible explanation, probably more related to the existence of the junction interface. This last possibility has been examined in various experiments, but no convincing, reproducible proof or clear definition of the controllable experimental conditions which lead to this effect $^{36,37}$ have been offered.

\section{MESOSCOPIC EFFECTS AND COHERENCE IN HTS NANOSTRUCTURE}

Nanotechnology can provide another way to study coherence and quasiparticle relaxation processes in HTS. The ultimate limit of GB performance in terms of yield and reproducibility, may be achieved when the junction dimensions get closer to the characteristic scaling lengths of HTS (i.e., coherence length, charge domains, and so on) and to the 
typical size of GB facets, which are one of the main sources of the lack of uniformity in the transport properties of GBS junctions. Thus, nanoscale junctions may be capable of isolating the intrinsic features of HTS systems, and turn out to be an ideal tool for better addressing the interesting topic of coherence in strongly correlated $d$-wave superconductors. These benefits will obviously extend to applications based on HTS junctions.

The first studies of bicrystal submicron JJs yielded encouraging results on the reduction of decoherence, ${ }^{53}$ the existence of a $2 \varphi$ component, ${ }^{54}$ and Andreev bound states. ${ }^{55}$

Recently, submicron biepitaxial junctions have been fabricated down to about $500 \mathrm{~nm}$ by $e$-beam lithography and by $\mathrm{C}$ and Ti masking. ${ }^{56}$ This step is even more significant because it has been applied to off-axis biepitaxial junctions, which manifest macroscopic quantum effects and are sensitive to directional transport along the lobes or the nodes of the $d$-wave OPS. Yield and reproducibility have been improved on this width scale. These improvements reflect advances in patterning simple nanobridges, which have been reproducibly scaled on $c$-axis YBCO down to about $100 \mathrm{~nm} .^{57}$ Flux dynamics has also been studied in nanorings with inner and external radii of about $150 \mathrm{~nm}$ and $300 \mathrm{~nm}$, respectively. ${ }^{58}$

This classical controllable "top-down" approach will be accompanied by some sort of "bottom-up" techniques based on the intrinsic nature of GB. The complex growth process may determine self-assembled nanochannels of variable dimensions, ranging typically from $20 \mathrm{~nm}$ to $200 \mathrm{~nm}$, often "enclosed" in macroscopic impurities. Even if this last technique is not ideal over the long range for applications (since it needs an additional critical step to locate the nanobridges and etch the HTS thin film), it can be really helpful for understanding the ultimate limits of the junction capabilities and understanding the transport mechanisms.

One example of the use of natural self-assembly to extract information on the physics of HTS Josephson junctions, is a study of universal conductance fluctuations (UCF) in magnetic fields in YBCO biepitaxial Josephson junctions. This is of relevance to the study of coherent quantum behavior in HTS. ${ }^{59,60}$ Structural studies make it possible, first, to locate macroscopic impurities which surround the conducting channel, whose size is roughly confirmed by the period of the magnetic pattern of the critical current. At low temperatures, quantum coherence can be monitored through the conductance $G$ of a normal metallic sample of length $L_{x}$ attached to two reservoirs. ${ }^{61,62}$ The electron wave packets that carry current in a diffusive wire have minimum size of the order of $L_{T}>L_{x} \gg l$. Here $l$ is the electron mean free path in the wire and $L_{T}$ is the thermal diffusion length ( $D$ is the diffusion constant). The first inequality is satisfied at relatively low temperatures such that $k_{B} T \ll \varepsilon_{C} \approx \hbar D / L_{x}^{2}$, where $\varepsilon_{C}$ is the Thouless energy. Conductance fluctuations become appreciable at low temperatures, over the entire range of magnetic fields. At low voltages $\left(e V \ll \varepsilon_{C}\right)$, the system is in a regime of universal conductance fluctuations: the variance $\left\langle g^{2}\right\rangle$ of the dimensionless conductance $g=G /\left(2 e^{2} / \hbar\right)$ is on the order of unity. The nonperiodic fluctuations have all the typical characteristics of mesoscopic fluctuations. ${ }^{61,62}$ Studies have been made at different voltages and under non- equilibrium conditions. An energy scale on the order of $1 \mathrm{meV}$ arises naturally from an analysis of the autocorrelation function of the conductance as a function of voltage. ${ }^{59,60}$ This has been identified as the Thouless energy $\varepsilon_{C}$, and its value is consistent with a channel size on the order of $100 \mathrm{~nm}$. This is proportional to the reciprocal of the time an electron spends in moving coherently across a mesoscopic sample. Quasiparticles seem to travel coherently across the junction even if $V \gg \varepsilon_{C}$. Hence, microscopic features of the weak link appear as less relevant, than mesoscopic, non local properties. In this case, the quasiparticle phase coherence time $\tau_{\varphi}$ does not seem to be limited by energy relaxation owing to a voltage-induced nonequilibrium. The remarkably long lifetime of the carriers found in these experiments, appears to be a generic property in high- $T_{C}$ YBCO junctions as demonstrated by optical measurements ${ }^{63}$ and macroscopic quantum tunneling. ${ }^{30,31}$

\section{CONCLUSIONS}

The results reviewed here are of great interest because they combine the stimulating subject of macroscopic quantum phenomena (MQT, ELQ) with new and important clues that such phenomena underlie the physics of HTS. We have focused on macroscopic quantum decay phenomena, as one of the most exciting manifestations of the Josephson effect. A system which displays macroscopic quantum effects despite the presence of nodes in the order parameter symmetry and therefore of low energy quasiparticles, raises several challenging questions about dissipation mechanisms and the particular coherence phenomena taking place in Josephson systems and HTS. We believe that advances in quantum engineering and nanotechnologies will stimulate further study of the Josephson effect and macroscopic quantum phenomena. Once the true "intrinsic" transport channels across junctions can be controlled and separated from the "extrinsic" contributions resulting from the complex morphology of the junctions (faceting, etc.), we should be able to get closer to the basic features of HTS (possibly stripes, spin-charge separation,...), but also have a more complete picture of the Josephson effect, and of the similarities and differences between HTS and LTS JJs. ${ }^{1-5}$

We acknowledge support from the EC STREP Project MIDAS-Macroscopic Interference Devices for Atomic and Solid State Physics: Quantum Control of Supercurrents.

We would like to thank Thilo Bauch, John R. Kirtley, Daniela Stornaiuolo and Arturo Tagliacozzo for valuable discussions.

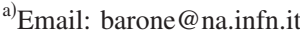

\footnotetext{
${ }^{1}$ B. D. Josephson, Phys. Lett. 1, 251 (1962).

${ }^{2}$ P. W. Anderson, Special Effects in Superconductivity, in: Lectures on the Manybody Problem, E. R. Caianiello (ed.), Vol. II p. 113, Academic Press, Ravello (1963).

${ }^{3}$ I. O. Kulik and K. Yanson, The Josephson Effect in Superconductive Tunneling Structures (Israel Program of Scientific Translations), Jerusalem (1972).

${ }^{4}$ A. Barone and G. Paternó, Physics and Applications of the Josephson Effect, Wiley, New York (1982).

${ }^{5}$ K. K. Likharev, Dynamics of Josephson Junctions and Circuits, Gordon
} 
and Breach, New York (1986); Rev. Mod. Phys. 51, 101 (1979).

${ }^{6}$ A. Barone, Weakly Coupled Macroscopic Quantum Systems: Likeness with Difference, I. O. Kulik and R. Ellialtioglu (eds.), Kluwer Academic Publishers (2000), p. 301.

${ }^{7}$ D. J. Van Harlingen, Rev. Mod. Phys. 67, 515 (1995).

${ }^{8}$ C. C. Tsuei and J. R. Kirtley, Rev. Mod. Phys. 72, 969 (2000) and Refs. cited therein.

${ }^{9}$ S. Coleman, Phys. Rev. D 15, 2929 (1977).

${ }^{10}$ A. J. Leggett, Macroscopic Quantum Systems and the Quantum Theory of Measurements, Suppl. Prog. Theor. Phys. 69, 80 (1980); A. J. Leggett, Quantum Tunneling of a Macroscopic Variable in Quantum Tunneling in Condensed Media, Yu. Kagan and A. J. Leggett (eds.), Elsevier Science Publ. (1992) and Refs. cited therein.

${ }^{11}$ Yu. M. Ivanchenko and L. A. Zilberman, Sov. Phys. JETP 28, 1272 (1969).

${ }^{12}$ A. O. Caldeira and A. J. Leggett, Phys. Rev. Lett. 46, 211 (1981).

${ }^{13}$ H. Grabert and U. Weiss, Phys. Rev. Lett. 53, 1787 (1984).

${ }^{14}$ A. I. Larkin and Yu. N. Ovchinnikov, Zh. Eksp. Teor. Fiz. 85, 1510 (1983) [Sov. Phys. JETP 58, 876 (1983)]; Phys. Rev. B 28, 828 (1983).

${ }^{15}$ A. Barone and Yu. N. Ovchinnikov, J. Low Temp. Phys. 55, 297 (1984); Yu. N. Ovchinnikov and A. Barone, J. Low Temp. Phys. 67, 323 (1987); A. Barone, J. Supercond: Incorporating Novel Magnetism 17, 585 (2004). ${ }^{16}$ R. F. Voss and R. A. Webb, Phys. Rev. Lett. 47, 265 (1981); L. D. Jackel, J. P. Gordon, E. L. Hu, R. E. Howard, L. A. Fetter, D. M. Tennant, R. W. Epworth, and J. Kurkijarvi, Phys. Rev. Lett. 47, 697 (1981); S. Washburn, R. A. Webb, R. F. Voss, and S. M. Farris, Phys. Rev. Lett. 54, 2712 (1985); D. B. Shwartz, B. Sen, C. N. Archie, and J. E. Lukens, Phys. Rev. Lett. 54, 1547 (1985); M. H. Devoret, J. M. Martinis, and J. Clarke, Phys. Rev. Lett. 55, 1908 (1985); W. der Boer and R. de Bruyn Ouboter, Physica B 98, 185 (1980); D. W. Bol, R. van Weelderen, and R. de Bruyn Ouboter, Physica B 122, 2 (1983); D. W. Bol, J. J. F. Scheffer, W. Giele, and R. de Bruyn Ouboter, Physica B 113, 196 (1985); R. J. Prance, A. P. Long, T. D. Clarke, A. Widom, J. E. Mutton, J. Sacco, M. W. Potts, G. Negaloudis, and F. Goodall, Nature 289, 543 (1981); I. M. Dimitrenko, V. A. Khlus, G. M. Tsoi, and V. I. Shnyrkov, Fiz. Nizk. Temp. 11, 146 (1985) [Sov. J. Low Temp. Phys. 11, 77 (1985)]; J. M. Martinis, M. H. Devoret, and J. Clarke, Phys. Rev. B 35, 4682 (1987); U. Weiss, H. Grabert, and S. Linkwitz, J. Low Temp. Phys. 68, 213 (1987).

${ }^{17}$ Josepshon Effect-Achievements and Trends ISI-85 Torino, A. Barone (ed.) World Scientific (1985) and Refs. reported therein.

${ }^{18}$ A. J. Leggett, S. Chakravarty, A. T. Dorsey, M. P. A. Fischer, A. Garg, and W. Zwerger, Rev. Mod. Phys. 59, 1 (1987).

${ }^{19}$ J. Clarke, A. N. Cleland, M. H. Devoret, D. Esteve, and J. M. Martinis, Science 29, 992 (1988).

${ }^{20}$ A. V. Zhuravlev and A. B. Zorin, Fiz. Nizk. Temp. 16, 184 (1990) [Sov. J. Low Temp. Phys. 16, 102 (1990)]; J. M. Schmidt, A. N. Cleland, and J. Clarke, Phys. Rev. B 43, 229 (1991).

${ }^{21}$ R. Rouse, S. Han, and J. E. Lukens, Phys. Rev. B B75, 1614 (1995).

${ }^{22}$ C. Cosmelli, P. Carelli, M. G. Castellano, F. Chiariello, G. D. Palazzi, R. Leoni, and G. Torrioli, Phys. Rev. Lett. 82, 5357 (1999). For aspects of the sample preparation see also M. G. Castellano et al., J. Appl. Phys. 80, 2928 (1996).

${ }^{23}$ Y. Nakamura, Y. Pashkin, and J. S. Tsai, Nature 398, 786 (1999).

${ }^{24}$ J. R. Friedman, V. Patel, W. Chen, S. K. Tolpygo, and J. E. Lukens, Nature 406, 43 (2000).

${ }^{25}$ L. B. Ioffe, V. B. Geshkenbein, M. V. Feigel'man, A. L. Fauchere, and G. Blatter, Nature 398, 679 (1999); C. C. Tsuei and J. R. Kirtley, Physica C (Amsterdam) 367, 1 (2002); B. Doucot, M. V. Feigel'man, and L. B. Ioffe, Phys. Rev. Lett. 90, 107003 (2003).

${ }^{26}$ A. Yu. Kitaev, preprint quant-ph/9707021 at xxx.lanl.gov, (1997).

${ }^{27}$ G. Blatter, V. B. Geshkenbein, and L. B. Ioffe, Phys. Rev. B 63, 174511 (2001).

${ }^{28}$ A. Wallraff, A. Lukashenko, J. Lisenfeld, A. Kemp, M. V. Fistul, Y. Koval, and A. V. Ustinov, Nature 425, 155 (2003).

${ }^{29}$ A. Kemp, A. Wallraff, and A. V. Ustinov, Phys. Status Solidi 233, 472 (2002).

${ }^{30}$ T. Bauch, F. Lombardi, F. Tafuri, G. Rotoli, A. Barone, P. Delsing, and T. Cleason, Phys. Rev. Lett. 94, 087003 (2005).

${ }^{31}$ T. Bauch, T. Lindström, F. Tafuri, G. Rotoli, P. Delsing, T. Claeson, and F. Lombardi, Science 311, 5757 (2006).

${ }^{32}$ Y. V. Fominov, A. A. Golubov, and M. Y. Kupriyanov, JETP Lett. 77, 587 (2003).

${ }^{33}$ M. H. S. Amin and A. Y. Smirnov, Phys. Rev. Lett. 92, 017001 (2004).

${ }^{34}$ S. Kawabata, S. Kashiwaya, Y. Asano, and Y. Tanaka, Phys. Rev. B 70, 132505 (2004).

${ }^{35}$ S. Kawabata, S. Kashiwaya, Y. Asano, and Y. Tanaka, Phys. Rev. B 72,
052506 (2005); T. Yokoyama, S. Kawabata, T. Kato, and Y. Tanaka, Phys. Rev. B 76, 134501 (2007).

${ }^{36}$ H. Hilgenkamp and J. Mannhart, Rev. Mod. Phys. 74, 485 (2002).

${ }^{37}$ F. Tafuri and J. R. Kirtley, Rep. Prog. Phys. 68, 2573 (2005).

${ }^{38}$ K. Inomata, S. Sato, K. Nakajima, A. Tanaka, Y. Takano, H. B. Wang, M. Nagao, H. Hatano, and S. Kawabata, Phys. Rev. Lett. 95, 107005 (2005).

${ }^{39}$ X. Y. Jin, J. Lisenfeld, Y. Koval, A. Lukashenko, A. V. Ustinov, and P. Mueller, Phys. Rev. Lett. 96, 177003 (2006).

${ }^{40}$ F. Tafuri, F. Miletto Granozio, F. Carillo, A. Di Chiara, K. Verbist, and G. Van Tendeloo, Phys. Rev. B 59, 11523 (1999).

${ }^{41}$ F. Lombardi, F. Tafuri, F. Ricci, F. Miletto Granozio, A. Barone, G. Testa, E. Sarnelli, J. R. Kirtley, and C. C. Tsuei, Phys. Rev. Lett. 89, 207001 (2002).

${ }^{42}$ F. Miletto Granozio, U. Scotti di Uccio, F. Lombardi, F. Ricci, F. Bevilacqua, G. Ausanio, F. Carillo, and F. Tafuri, Phys. Rev. B 67, 184506 (2003).

${ }^{43}$ F. Tafuri, J. R. Kirtley, F. Lombardi, and F. Miletto Granozio, Phys. Rev. B 67, 174516 (2003).

${ }^{44}$ C.-R. Hu, Phys. Rev. Lett. 72, 1526 (1994); T. Löfwander, V. S. Shumeiko, and G. Wendin, Supercond. Sci. Technol. 14, R53 (2001); S. Kashiwaya and Y. Tanaka, Rep. Prog. Phys. 63, 1641 (2000).

${ }^{45}$ E. Ilichev, V. Zakosarenko, R. P. J. Ijsselsteijn, H. E. Hoenig, V. Schultze, H. G. Meyer, M. Grajcar, and R. Hlubina, Phys. Rev. B 60, 3096 (1999); E. E. Il'ichev, M. Grajcar, R. Hlubina, R. P. J. IJsselsteijn, H. E. Hoenig, H.-G. Meyer, A. Golubov, M. H. S. Amin, A. M. Zagoskin, A. N. Omelyanchouk, and M. Yu. Kupriyanov, Phys. Rev. Lett. 86, 5369 (2001); T. Lindstrom, S. A. Charlebois, A. Ya. Tzalenchuk, Z. Ivanov, M. H. S. Amin, and A. M. Zagoskin, Phys. Rev. Lett. 90, 117002 (2003).

${ }^{46}$ The dynamics of a JJ can be described by assuming the presence of both first and second harmonic components in the CPR (higher harmonics can be neglected because of low junction barrier transparency). Here $I$ $=I_{1}(\sin \phi-a \sin 2 \phi)$ where $a=I_{2} / I_{1}$, and $I_{2}$ and $I_{1}$ are the second and first harmonic components. For a given value of a, the dynamics is equivalent ${ }^{47}$ to that of a particle of mass $\mathrm{m}_{\varphi}$ moving in a washboard potential $U(a)=$ $-\left(I_{1} \Phi_{0} / 2 \pi\right)\left(\left(I_{C O} / I_{1}\right) \gamma \phi+\cos -(a / 2) \cos 2 \phi\right)$, where $E_{1}=I_{1} \Phi_{0} / 2 \pi$ is the first harmonic of the Josephson energy and $\Phi_{0}$ is the quantum of flux. The normalized bias current $\gamma=I / I_{C} 0$ determines the tilt of the potential, where $I_{C O}=I_{1} \max (\sin \phi-a \sin 2 \phi)$ is the maximum critical current of the JJ. For zero bias and $a>0.5$ the potential has the shape of a double well. When $0.5<a<2$ the phase will always undergo a transition into the running (moving) state from the lower lying well of the tilted double-welled washboard potential; this can be confirmed by numerical simulations of the Langevin equation of motion. More details can be found in A. Ya. Tzalenchuk, T. Lindström, S. A. Charlebois, E. A. Stepantsov, Z. Ivanov, and A. M. Zagoskin, Phys. Rev. B 68, 100501 (2003).

${ }^{47}$ W. C. Stewart, Appl. Phys. Lett. 12, 277 (1968); D. E. McCumber, J. Appl. Phys. 39, 3113 (1968).

${ }^{48}$ S. Kawabata, T. Bauch, and T. Kato, Phys. Rev. B 80, 174513 (2009).

${ }^{49}$ L. D. Jackel, J. P. Gordon, E. L. Hu, R. E. Howard, L. A. Fetter, D. M. Tennant, R. W. Epworth, and J. Kurkijärvi, Phys. Rev. Lett. 47, 697 (1981).

${ }^{50}$ F. Balestro, J. Claudon, J. P. Pekola, and O. Buisson, Phys. Rev. Lett. 91, 158301 (2003).

${ }^{51}$ G. Rotoli, T. Bauch, T. Lindström, D. Stornaiuolo, F. Tafuri, and F. Lombardi, Phys. Rev. B 75, 144501 (2007).

${ }^{52}$ X. G. Wen and P. A. Lee, Phys. Rev. Lett. 80, 2193 (1998).

${ }^{53}$ A. Ya. Tzalenchuk, T. Lindström, S. A. Charlebois, E. A. Stepantsov, Z. Ivanov, and A. M. Zagoskin, Phys. Rev. B 68, 100501 (2003).

${ }^{54}$ E. Il'ichev, M. Grajcar, R. Hlubina, R. P. J. IJsselsteijn, H. E. Hoenig, H.-G. Meyer, A. Golubov, M. H. S. Amin, A. M. Zagoskin, A. N. Omelyanchouk, and M. Yu. Kupriyanov, Phys. Rev. Lett. 86, 5369 (2001).

${ }^{55}$ G. Testa, A. Monaco, E. Esposito, E. Sarnelli, D. J. Kang, S. H. Mennema, E. J. Tarte, and M. G. Blamire, Appl. Phys. Lett. 85, 1202 (2004); G. Testa, E. Sarnelli, A. Monaco, E. Esposito, M. Ejrnaes, D. J. Kang, S. H. Mennema, E. J. Tarte, and M. G. Blamire, Phys. Rev. B 71, 134520 (2005).

${ }^{56}$ D. Stornaiuolo, G. Rotoli, K. Cedergen, T. Bauch, F. Lombardi, and F. Tafuri, to be published in J. Appl. Phys. (2010); D. Stornaiuolo, E. Gambale, T. Bauch, D. Born, K. Cedergren, D. Dalena, A. Barone, A. Tagliacozzo, F. Lombardi, and F. Tafuri, Physica C 468, 310 (2008).

${ }^{57}$ G. Papari, F. Carillo, D. Born, L. Bartoloni, E. Gambale, D. Stornaiuolo, P. Pingue, F. Beltram, and F. Tafuri, IEEE Trans. Appl. Supercond. 19, 183 (2009).

${ }^{58}$ F. Carillo, G. Papari, D. Stornaiuolo, D. Born, D. Montemurro, P. Pingue, F. Beltram, and F. Tafuri, Phys. Rev. B 81, 054505 (2010).

${ }^{59}$ A. Tagliacozzo, D. Born, D. Stornaiuolo, E. Gambale, D. Dalena, F. Lom- 
bardi, A. Barone, B. L. Altshuler, and F. Tafuri, Phys. Rev. B 75, 012507 (2007).

${ }^{60}$ A. Tagliacozzo, F. Tafuri, E. Gambale, B. Jouault, D. Born, P. Lucignano, D. Stornaiuolo, F. Lombardi, A. Barone, and B. L. Altshuler, Phys. Rev. B 79, 024501 (2009).

${ }^{61}$ B. L. Altshuler and A. G. Aronov, in: Electron-Electron Interaction in Disordered Systems, A. L. Efros and M. Pollak Elsevier (eds.), Amsterdam (1985); P. A. Lee and T. V. Ramakrishnan, Rev. Mod. Phys. 57, 287 (1985); P. A. Lee and A. D. Stone, Phys. Rev. Lett. 55, 1622 (1985); P. A. Lee, A. D. Stone, and H. Fukuyama, Phys. Rev. B 35, 1039 (1987); B. Al'tshuler and P. Lee, Phys. Today 41, 36 (1988); R. A. Webb and S.
Washburn, Phys. Today 41, 46 (1988); For a review see Mesoscopic Phenomena in Solids, B. L. Altshuler, P. A. Lee, and R. A. Webb (eds.), North-Holland, New York, (1991).

${ }^{62}$ Y. Imry, Introduction to Mesoscopic Physics, Oxford University Press (1997).

${ }^{63}$ N. Gedik, J. Orenstein, R. Liang, D. A. Bonn, and W. N. Hardy, Science 300, 1410 (2003).

This article was published in English in the original Russian journal. Reproduced here with stylistic changes by AIP. 